\title{
Observations sur un essai préliminaire de lâchers de mâles stériles de Glossina palpalis gambiensis (Haute-Volta)
}

\author{
par M. ClaiR $(*)$, H. POl.ITZAR $\left({ }^{*}\right)$, D. CUISANCE $\left(^{*}\right)$ et A. LAFAYE $\left(^{* *}\right)$ \\ (avec la collaboration technique de E. SELLIN)
}

\begin{abstract}
RĚSUMÉ
Durant la saison des pluies 1975,5556 mâles de G. p. gambiensis, irradiés à 15000 rads, ont été lâchés pendant 4 mo1s, au cours de 25 séances, dans un bois sacré proche de Bobo-Dioulasso. Les pertes enregistrées à l'envol sont faibles $(6,7$ p. 100). Des sondages réguliers au moyen du piège Challier indiquent un rapport moyen de 1 mâle stérile pour 1 mâle sauvage pendant cette période d'observation, fluctuant suivant les quantités lảchées et selon l'évolution de la densité naturelle du gîte.

La dispersion est bonne dès le $4^{c}$ jour suivant le lâcher et la longévité 50 p. 100 est de 6,2 jours tandis que la longévité maximale enregistrée est en moyenne de 23 jours. Les mâles ains1 lâchés, dont le nombre est limité et qui subissent une forte dose stérilisante, ont cependant provoqué une absence de larviposition chez 25 p. 100 des femelles sauvages mises en observation après capture.

Cet essai préliminaire a permis d'étudier le comportement de mâles stériles de l'espèce $G$. palpalis gambiensis lâchés sur le terrain et de roder matériel et personnel avant des làchers prochains à plus grande échelle.
\end{abstract}

\section{INTRODUCTION}

En 1974, un projet de lutte contre Glossina palpalis gambiensis Vanderplank, 1949, par lâchers de mâles irradiés a commencé à BoboDioulasso (Haute-Volta) (2). La première étape de cette expérimentation a consisté à installer au laboratoire un élevage de cette espèce. Celuici a débuté en janvier 1975 ; il est prévu un effectif total de 30000 femelles, ce qui ne peut être obtenu qu'après plusieurs mois. De ce fait, de nombreux mâles éclos dans l'insectarium ne sont pas conservés afin d'éviter la surcharge qui pèserait alors sur l'effectif de lapins, hôtes nour-

(*) Centre I. E. M. V. T. de Recherches sur les Trypanosomiases Animales, B. P. 454, Bobo-Dioulasso (République de Haute-Volta).

(**) Centre de Documentation et de Statistique de l'O. C. C. G. E., B. P. 153, Bobo-Dioulasso (République de Haute-Volta). riciers de ces glossines. Seules les femelles reproductrices sont gardées pour l'élevage ainsi qu'un lot de mâles étalons. Ce surplus d'individus mâles a été utilisé pour effectuer, après irradiation et lâchers sur le terrain, une série d'observations dont les résultats sont présentés ici.

\section{LIEU D'OBSERVATION}

\section{Situation (schéma $\mathrm{n}^{\circ}$ 1)}

Le gîte retenu est le bois sacré de San, situé à $9 \mathrm{~km}$ de Bobo-Dioulasso, au bord de l'axe routier qui conduit à Ouagadougou. Un petit ruisseau, le Poa, prend naissance dans la forêt classée du même nom sur le «plateau de Bobo » où son cours, en pente faible, franchit, après quelques centaines de mètres, un seuil rocheux très marqué, la falaise dite "de Banfora» 


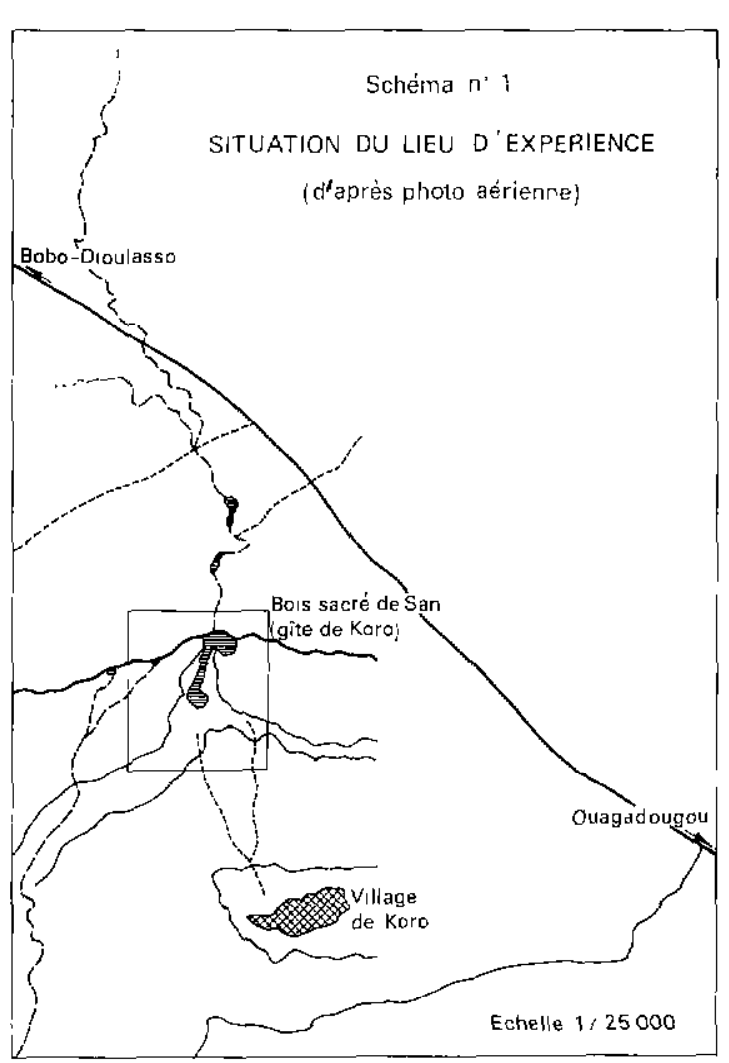

constituant une chute d'une quinzaine de mètres. C'est un affluent de la Koba qui se jette dans la Bougouriba, cette dernière rejoignant la Volta Noire.

\section{Aspect (schéma $n^{\circ}$ 2)}

Au pied de cette falaise se trouve le bois sacré de San. Installé au milieu d'un énorme éboulis rocheux, il s'étend sur $50 \mathrm{~m}$ de large et $100 \mathrm{~m}$ de long et entoure la cascade. Il s'étire ensuite le long du petit ruisseau pour former une sorte de galerie forestière plus ou moins régulière et se termine après $200 \mathrm{~m}$ environ dans des plantations de manguiers et de bananiers que cultivent les habitants du village de Koro, dont ce site constitue un lieu sacré.

Sur « le plateau de Bobo" le cours de ce ruisseau est temporaire. Une résurgence à mihauteur de la falaise assure un débit très faible mais permanent dans le cours inférieur.

Ce biotope assez pittoresque constitue un gîte permanent de G. p. gambiensis qui est la seule espèce représentée.

Au pied de la cascade la végétation est luxuriante, constituée principalement de grands arbres (Ficus sp., Berlinia sp., Vitex sp.) et de palmiers (Elaeis guineensis) que recouvrent des plantes lianescentes. Le long du ruisseau, la végétation ripicole est surtout arbustive et buissonnante.

Les manguiers assez groupés prolongent artificiellement ce gîte dont la longueur totale est de $350 \mathrm{~m}$ environ.

Autour de ce lieu, toute végétation dense a disparu pour laisser place à des champs (mil, maïs, igname) et à une savane herbeuse. La dégradation de la végétation riveraine en amont de la falaise et la mise en culture en aval font du bois de San un gîte totalement isolé.

\section{Hôtes nourriciers}

L'homme qui vient cultiver ou pêcher est certainement l'hôte nourricier principal de G. p. gambiensis dans ce biotope. Cependant, le caractère sacré du site permet à une faune de se maintenir encore : varans, serpents, singes et quelques petites antilopes.

\section{ORIGINE DES MALES STÉRILES}

\section{Elevage}

Celui-ci a pour origine un lot de 5000 pupes provenant de l'élevage de G. p. gambiensis réalisé depuis 1972 au Laboratoire d'Entomologie de l'I. E. M. V. T. à Maisons-Alfort (9). G. p. gambiensis est élevée au Centre I. E. M. V. T. de Bobo-Dioulasso dans des salles climatisées, maintenues à une température de $25^{\circ} \mathrm{C}$ et à une humidité de 75 à 85 p. 100 de façon continue.

Les mâles âgés de 6 jours sont accouplés à des femelles de trois jours pendant 48 heures puis séparés.

Les femelles regroupées par cages de 25 individus sont alors stockées sur des pondoirs dont la production journalière est rassemblée par unité de 1000. A l'éclosion, mâles et femelles sont séparés après immobilisation par le froid à $+4^{\circ}$. L'alimentation se fait sur oreilles de lapin. L'âge des mâles lâchés varie de 1 à 3 jours.

\section{Stérilisation}

\subsection{Appareil}

Le laboratoire est équipé d'un irradiateur autonome monobloc G. A. A. A. $30(*)$, com-

(*) Groupement pour les Activités Atomiques et Avancées, 20, av. Edouard-Herriot, 92350 Le PlessisRobinson (France). 


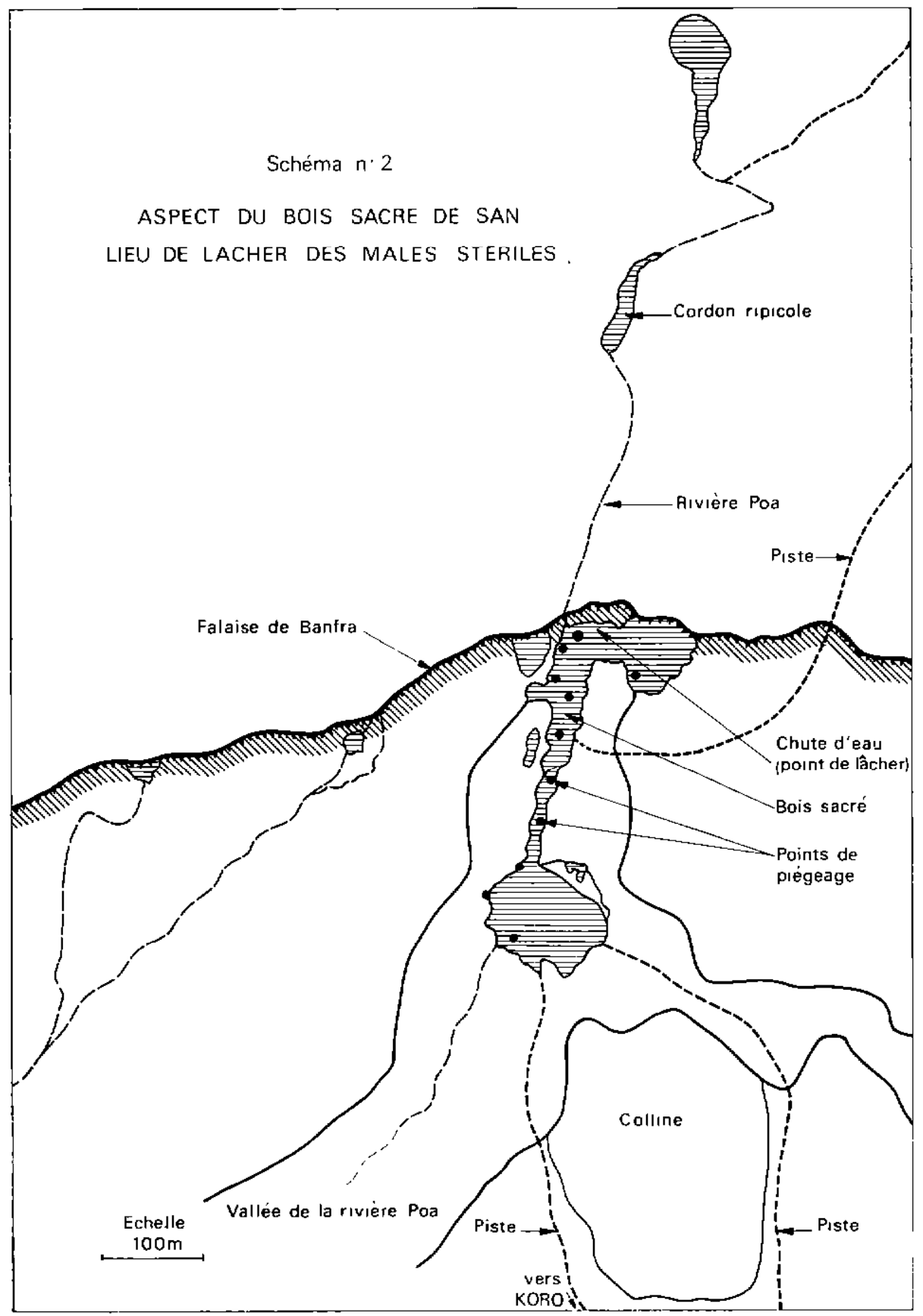

Rectificatif : lire Falaise de Banfora.

portant une chambre d'irradiation de $30 \mathrm{dm}^{3}$, dans une enceinte en plomb, irradiée par quatre sources radio-actives (Cæsium 137), d'une puissance totale de 12400 curies, donnant un débit de dose de $63000 \mathrm{rads} /$ heure $( \pm 12$ p. 100. Une minuterie avec alarme sonore permet le décompte du temps d'irradiation.

\subsection{Doses}

Les quantités relativement réduites de mâles disponibles au début de l'élevage entrepris à
Bobo-Dioulasso n'ont permis que des essais limités concernant d'une part le bon fonctionnement de l'irradiateur et sa fiabilité et d'autre part l'appréciation de la dose optimale d'irradiation des mâles.

Des adultes âgés de 1 jour et de 3 jours sont irradiés à 8000,12000 et 15000 rads. Après ce traitement, les mâles sont accouplés à un lot numériquement identique de femelles vierges dont la production de pupes est notée ; 15 jours 
TABL. $N^{\bullet} I$-Production de pupes par des femelles accouplées à des mâles irradiés.

\begin{tabular}{|l|c|c|c|c|c|c|c|c|}
\hline & \multirow{2}{*}{ Age } & \multirow{2}{*}{$\begin{array}{c}\text { Doses (rads) } \\
\text { irradiation }\end{array}$} & $\begin{array}{c}\text { Nombre } \\
\sigma\end{array}$ & $\begin{array}{c}\text { Nombre } \\
\text { q }\end{array}$ & $\begin{array}{c}\text { Nombre } \\
\text { pupes }\end{array}$ & $\begin{array}{c}\text { Nombre o } \\
\text { restants }\end{array}$ & $\begin{array}{c}\text { Nombre } \\
\text { accouplées }\end{array}$ & $\begin{array}{c}\text { Nombre } \\
\text { pupes }\end{array}$ \\
\hline Témoins & 1 jour & 0 & 8 & 8 & 50 & - & - & - \\
\hline \multirow{5}{*}{ Irradiës } & 1 jour & 8000 & 20 & 20 & 13 & 8 & 8 & 1 \\
& 1 jour & 12000 & 10 & 8 & 4 & 5 & 5 & 0 \\
& 3 jours & 8000 & 10 & 10 & 14 & 9 & 9 & 8 \\
& 3 jours & 15000 & 10 & 9 & 0 & 8 & 8 & 0 \\
\hline
\end{tabular}

après, un nouvel accouplement a lieu avec un second lot de femelles vierges dont la fertilité est également suivie. Le tableau ci-dessous indique les résultats obtenus avec des mâles irradiés à différentes doses comparativement à un lot témoin, pendant une durée d'observation de 3 mois.

Les résultats (tabl. I) montrent qu'à 8000 rads le taux de stérilité est élevé, mais encore insuffisant puisque le premier comme le deuxième accouplement de mâles irradiés avec des femelles vierges sont susceptibles d'être en partie féconds.

La dose de 15000 rads donne par contre une stérilité totale au premier comme au deuxième accouplement.

Les quantités d'individus disponibles étant limitées, un testage de doses intermédiaires n'est pas réalisé à ce moment, si bien que les mâles lâchés dans le bois de San sont irradiés à la dose stérilisante de 15000 rads.

Des lots plus représentatifs de mâles (quarante chacun) ont cependant été mis en observation à la suite d'un envoi de pupes de MaisonsAlfort, les doses d'irradiation étant alors de $10000,11000,12000,13000$ et 14000 rads. Mais les conditions défectueuses du transport entre Maisons-Alfort et Bobo-Dioulasso n'ont pas permis de tirer de conclusions valables de cette série d'irradiation. Les données d'un essai récent viendront prochainement compléter ces observations préliminaires en rendant compte de la stérilité et de la compétitivité obtenue.

\section{Transport}

L'acheminement des lots de mâles stériles se fait en véhicule jusqu'à la falaise puis à pied jusqu'au bois de San. Les cages sont enfermées dans une boite isotherme pendant la durée du transport qui n'excède pas une demi-heure.

\section{LACHERS DES MALES STÉRILES}

\section{Marquage}

Suivant une technique déjà utilisée $(3,4,5,6)$, une tache de gouache acrylique est déposée sur le thorax de la glossine. Cette méthode simple et facile donne de bons résultats. Une gamme de différents coloris est utilisée : blanc, jaune, rouge, vert. La combinaison de ces derniers entre eux autorise une assez grande latitude de marquage.

\section{Bilan au moment du lâcher (tabl. II)}

Les lâchers ont eu lieu du 18 juillet au 19 novembre, soit pendant 4 mois, correspondant à la saison des pluies.

\begin{tabular}{|l|r|c|}
\hline \multicolumn{3}{|c|}{ TABLEAU $N^{\circ}$ II } \\
\hline Mâles stếriles & Nombre & p. 100 \\
\hline Ayant pris envol & 5556 & 93,3 \\
Tombês & 287 & 4,8 \\
Morts & 112 & 1,9 \\
\hline Total & 5955 & 100 \\
\hline
\end{tabular}

\subsection{Glossines ayant pris leur envol}

93,3 p. 100 prennent un envol normal après être libérées. Ce pourcentage est exceptionnellement bon malgré la forte dose d'irradiation reçue par ces individus qui subissent en outre diverses manipulations: refroidissement, marquage, transport.

\subsection{Glossines tombées et mortes}

6,7 p. 100 de pertes sont enregistrées dont 4,8 p. 100 d'individus trop faibles ou dont les ailes mal dépliées ou malformées ne permettent pas de prendre un envol normal.

Ce taux semble très correct et inférieur à ce que l'on pourrait attendre.

Ces bonnes performances sont imputables à 
un transport rapide durant une période climatique favorable (saison des pluies). Le temps bref qui sépare la stérilisation du moment du lâcher favorise également le bon envol des mâles stériles dans le bois de San.

\section{Points de lầcher}

La presque totalité des lâchers a lieu en un point unique, dit « point zéro » qui correspond au bas de la chute d'eau. En fin de saison sèchedébut de saison des pluies, période où commencent les lâchers, les captures régulières montrent que la population sauvage de glossines est surtout concentrée dans le $1 / 3$ supérieur du gîte. De ce fait, jusqu'à la fin août, les lâchers ont été effectués en ce lieu. A partir de septembre, la dispersion des glossines sauvages devient plus grande et les lâchers ont en conséquence été fractionnés; les mâles irradiés sont libérés à partie égale dans le $1 / 3$ supérieur et dans le 1/3 inférieur du gîte (groupe de manguiers et de bananiers).

\section{Nombre de mâles stériles lâchés : propor- tion mâles stériles/mâles sauvages}

\subsection{Fréquence des lâchers (tabl. III)}

De juillet à novembre, les mâles stériles sont lâchés au cours de 25 séances. Le surplus de mâles produit au laboratoire pendant cette période permet d'effectuer un lâcher par semaine durant les mois de juillet, août, septembre, deux lâchers par semaine en octobre et novembre.

\subsection{Rapport mâles stériles/mâles sauvages}

Des sondages hebdomadaires sont réalisés dans le gîte. La récolte des glossines se fait non plus par une équipe de captureurs mais au moyen d'un piège biconique conçu par CHALLIER et LAVEISSIERE (1). Dix pièges biconiques sont utilisés régulièrement pendant toute la durée des lâchers. Cette nouvelle technique permet des captures simultanées en différents points du gîte avec une efficacité au moins aussi bonne que celle des captureurs et une plus grande régu- larité. L'objet de ces sondages est d'apprécier et de suivre la proportion de mâles stériles vivant dans le gîte en fonction des lâchers. Deux essais d'estimation de ce paramètre sont réalisés.

\subsubsection{Essai d'évaluation du rapport mâles stériles/mâles sauvages au moment des lâchers}

Il est nécessaire pour évaluer ce rapport de connaître la densité réelle en glossines sauvages du gîte. La méthode de capture-marquagerecapture est utilisée durant le mois de juillet, mais est volontairement écartée ensuite pour éviter de trop perturber l'équilibre naturel du gîte par des marquages trop fréquents de glossines sauvages. Elle permet d'obtenir une valeur de la densité réelle qui est comparée à la densité apparente obtenue par les récoltes au piège.

Le rapport de l'une à l'autre ou disponibilité (availability) est de 7,5 en moyenne en juillet. On retient donc que, dans le bois de San, lorsqu'une glossine est capturée au piège, il en existe un peu plus de 7 dans le gite.

D'une part on connaît les quantités de mâles stériles qui sont lâchées, d'autre part des mâles stériles issus des précédents lâchers vivent encore dans le gitc dont un certain nombre sont repris au piège. La somme de tous ces mâles stériles est rapportée à la population de mâles sauvages du gîte (tabl. IV).

Cette méthode d'approche de la réalité indique que, en moyenne, il y a presque un mâle stérile pour un mâle sauvage (C,9:1) dans ce gîte durant les 4 mois de lâcher.

\subsubsection{Valeur du rapport mâles stériles/mâles sauvages d'après les résultats des cap- tures}

Les captures au piège ayant lieu une à deux fois par semaine, le nombre de mâles stériles et le nombre de mâles sauvages récoltés sont enregistrés. Le rapport $\varnothing \rightarrow / \delta$ a une valeur moyenne, pendant toute la durée de l'expérimentation de 1,3/1 (tabl. V).

TABL. $N^{\bullet}$ III-Fréquence des lâchers. Nombre de mâles stêriles lâchếs

\begin{tabular}{|l|c|c|c|c|c|}
\hline & Juillet & Août & Septembre & Octobre & Novembre \\
\hline $\begin{array}{l}\text { Nombre de séances } \\
\text { de lâchers }\end{array}$ & 3 & 5 & 4 & 8 & 5 \\
$\begin{array}{l}\text { Nombre de mâles } \\
\text { Etériles 1êchés }\end{array}$ & 832 & 645 & 822 & 256 & 1961 \\
\hline
\end{tabular}


TABL. N ${ }^{\circ}$ IV-Essai d'êvaluation du rapport : $0^{n}$ au moment des lâchers.

\begin{tabular}{|c|c|c|c|c|c|c|}
\hline Mois & $\begin{array}{c}\text { Densite } \\
\text { apparente } \delta\end{array}$ & $\begin{array}{l}\text { Densité } \\
\text { rĕel1e } \\
\tilde{a}(x 7,5)\end{array}$ & 1âchës & $\begin{array}{l}\text { "' restants } \\
\text { des précédents } \\
\text { lâchers }\end{array}$ & $\begin{array}{l}\text { Total } \\
\text { présents } \\
\text { dans gite }\end{array}$ & $\infty: 0$ \\
\hline Juillet & 104 & 780 & 832 & 133 & 965 & $1,2: 1$ \\
\hline Août & 177 & 1327 & 645 & 159 & 804 & $0,6: 1$ \\
\hline Septembre & 276 & 2070 & 822 & 206 & 1028 & $0,4: 1$ \\
\hline Octobre & 293 & 2197 & 2196 & 464 & 2560 & $1,1: 1$ \\
\hline Novembre & 102 & 765 & 1061 & 239 & 1300 & $1,6: 1$ \\
\hline \multicolumn{5}{|c|}{$0^{7}$ : mâles sauvages; $e^{*}$ mâles stêriles. } & moyenne: & $0,9: 1$ \\
\hline
\end{tabular}

Rectificatif, $5^{\mathrm{e}}$ colonne, lire $: \varnothing \rightarrow$ restants.

TABL. $N^{\circ} \mathrm{V}$-Valeur du rapport $\phi^{1}: 0^{-1}$ d'après les résultats des captures

\begin{tabular}{|l|c|c|c|}
\hline \multicolumn{1}{|c|}{ Mois } & $0^{2}$ capturés & capturés & $0^{7}$ \\
\hline Juillet & 242 & 432 & $2,0: 1$ \\
Août & 576 & 568 & $0,9: 1$ \\
Septembre & 787 & 734 & $0,9: 1$ \\
Octobre & 589 & 946 & $1,6: 1$ \\
Novembre & 246 & 587 & $2,3: 1$ \\
\hline & & moyenne : $1,3: 1$ \\
\hline
\end{tabular}

\subsubsection{Discussion}

Le rapport $\varnothing \rightarrow / \sigma^{*}$ obtenu par la première méthode est de $0,9: 1$; il est de $1,3: 1$ par la seconde. On peut admettre qu'il est légèrement sous-estimé dans le premier cas par le fait que le nombre de mâles stériles vivants dans le gîte à la suite des lâchers est plus important que ne l'indique ce calcul. Il est vraisemblablement un peu surestimé dans le second cas par le fait que les mâles stériles viennent plus facilement au piège que les mâles sauvages (moindre adaptation aux conditions naturelles les premiers jours).

On peut raisonnablement penser, en s'appuyant sur les renseignements fournis par ces 2 méthodes, que le rapport des mâles stériles aux mâles sauvages est, dans le bois de San, voisin de 1.

\subsection{Evolution de ce rapport}

Durant la période d'observation, qui correspond à la saison des pluies, on note que ce rapport varie (graphique 1).

- Il baisse en juillet, août et septembre : les

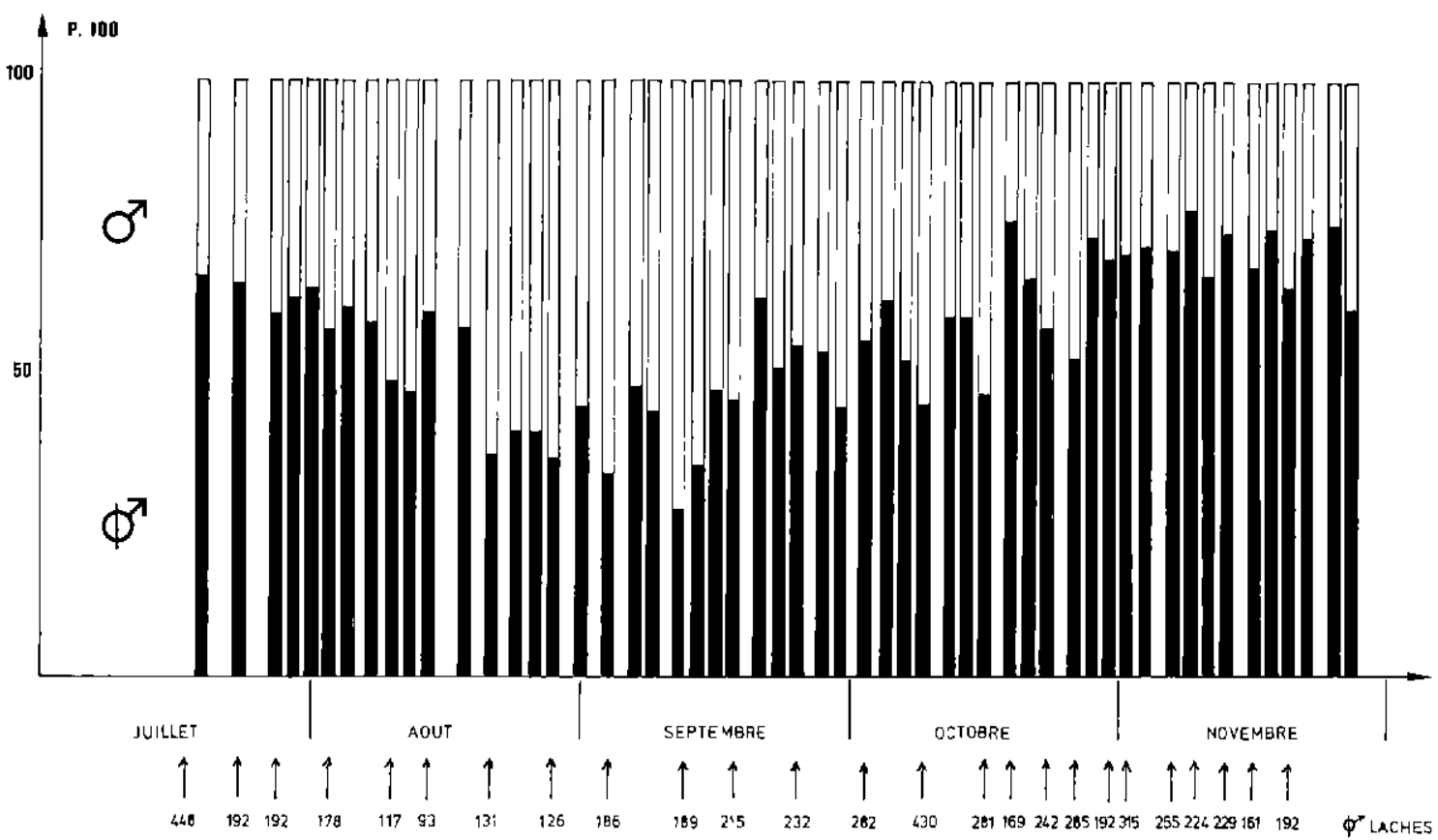

Pourcentage de mâles stériles au cours des captures en fonction des lâchers. 
quantités de mâles stériles lâchés sont restées sensiblement stationnaires à cette époque tandis que la densité de la population sauvage du gîte triple. Le rapport $\varnothing \rightarrow / \delta$ passe alors de 1,2 à 0,4 .

- En octobre, la densité de la population sauvage reste encore élevée mais les lâchers devenant quantitativement importants et rapprochés dans le temps ( 2 fois/semaine), le rapport s'améliore à l'avantage des mâles stériles $(1,1: 1)$ et celui-ci devient nettement favorable en novembre $(1,6: 1)$, période où la densité naturelle baisse.

\section{Quelques aspects du comportement des mâles stériles lâchés}

\subsection{Dispersion}

Pendant les deux premiers mois de l'observation, les lâchers ont lieu en un point unique au bas de la cascade. Dix pièges sont disposés à des intervalles de $30 \mathrm{~m}$ environ sur toute la longueur du gîte à partir de ce «point zéro ». Connaissant la position exacte des pièges par rapport au point de lâcher pris comme origine, il est facile de noter à chaque capture la distance parcourue par les mâles stériles.

Les distances moyennes parcourues par les mâles stériles dans les jours suivant les lâchers sont respectivement de :

$88,1 \mathrm{~m}( \pm 8,3)$ au délai de 2 jours;

99,8 $\mathrm{m}( \pm 13,3)$ au délai de 3 jours ;

$147,5 \mathrm{~m}( \pm 28,2)$ au délai de 4 jours.

Seuls trois lâchers de mâles stériles ont été accompagnés d'un lâcher de mâles et femelles sauvages marqués afin de comparer les deux lots. On retiendra dans ce cas les délais de 2 jours et 4 jours pour lesquels les effectifs capturés sont suffisamment grands.

Au délai de 2 jours, les mâles stériles atteignent alors en moyenne $89,4 \mathrm{~m}( \pm 10,2)$ tandis que les mâles et femelles sauvages franchissent 195,7 m ( $\pm 26,2)$. La différence est nettement significative $(\mathrm{t}=6,82 ;$ d. d. $1 .=164) .48$ heures après le lâcher, les mâles sauvages ont parcouru une plus grande distance que les mâles stériles.

Au délai de 4 jours, mâles stériles et glossines sauvages atteignent respectivement $147,5 \mathrm{~m}$ et $143,2 \mathrm{~m}$. II n'y a plus à ce délai de différence significative $(\mathrm{t}=0,10$; d. d. $1 .=64)$. Quatre jours après le lâcher, il semble donc que les mâles stériles soient aussi bien dispersés que les glossines sauvages. Cette performance apparaît meilleure que celle observée pour $G$. tachinoides au Tchad $(4,6)$ où il fallait 6 jours pour obtenir la même dispersion dans les 2 lots. Il faut vraisemblablement l'attribuer ici à la rapidité du transport et aux bonnes conditions climatiques de la saison humide.

\subsection{Longévité}

Les recaptures, par pièges, d'individus marqués fournissent des données sur les quantités d'individus en vie à des délais variables après leur lâcher. Sur un total de 21 lâchers, des échantillonnages réguliers sont réalisés toutes les semaines, pendant plus d'un mois en moyenne pour chacun d'eux.

\subsubsection{Durée de survie moyenne: longévité du groupe}

Il est possible d'estimer la survie moyenne dans le milieu extérieur, à partir des pourcentages de recapture, à l'aide de 2 méthodes complémentaires faisant intervenir les concepts de risque de capture et de risque de disparition.

5.2.1.1. Le risque de capture est déterminé en ajustant à la distribution des recaptures (taux quotidiens pondérés) un modèle exponentiel à taux instantané variable, loi GAMMA $(r=0,32$ $\mathrm{k}=2,55$ ) ou, surtout, loi de WEIBULL ( $\mathrm{B}=1,40, \eta=7,50 \gamma=0,50$ ) qui représentent de façon très satisfaisante les données observées. On en déduit que le risque de capture est croissant et que la médiane de la distribution (50 p. 100 de captures) est atteinte après un délai de 6,3-6,4 jours, compris dans l'intervalle 6-7 jours noté au simple examen des taux pondérés cumulés.

5.2.1.2. Le risque de disparition des mâles stériles dans le milieu extérieur est déterminé à partir de la courbe théorique de l'évolution des taux de recapture (régression parabolique calculée par les moindres carrés pondérés) sous l'hypothèse de proportionnalité entre ces taux et les effectifs encore présents. Le risque de disparition est également croissant. Il découle d'une table de «survie " élaborée à l'aide de taux de «survie" déterminés graphiquement sur papier semi-logarithmique ou par le calcul en considérant les différences entre taux de recapture successifs. D'après cette table, 50 p. 100 des mâles stériles ont disparu du milieu extérieur après un délai de 8 jours.

Bien que les deux risques considérés soient également croissants, il est intéressant de noter 
que l'accroissement du risque de capture est surtout marqué dans les premiers jours, contrairement au risque de disparition qui augmente considérablement dans les derniers jours de la « survie».

\subsubsection{Durée de survie maximale: longévité individuelle}

Elle représente la durée maximale après le lâcher au bout de laquelle il est possible de capturer encore un individu marqué. Sur 25 lâchers de mâles stériles, 21 donnent lieu à une observation régulière dans les semaines suivantes afin d'apprécier les records de survie. La moyenne ainsi obtenue de juillet à novembre est de 23 jours $\pm 2,2$ ( 1 cas à 35 jours).

$\mathrm{Si}$ on compare uniquement la moyenne obtenue pour les trois lots de mâles stériles lâchés en même temps que les trois lots de glossines sauvages au mois de juillet, les résultats sont les suivants :

$$
\begin{aligned}
& \text { mâles stériles } \varnothing \rightarrow: \\
& 28,2 \text { jours } \pm 3,4 \text { ( } 1 \text { cas à } 35 \text { jours }) \text {; } \\
& \text { mâles sauvages } \not \text { : } \\
& 38,5 \text { jours } \pm 7,8 \text { ( } 1 \text { cas à } 45 \text { jours }) \text {; } \\
& \text { femelles sauvages } \% \text { : } \\
& 37,0 \text { jours } \pm 16,8 \text { ( } 1 \text { cas à } 56 \text { jours }) \text {. }
\end{aligned}
$$

La différence entre les longévités maximales des mâles stériles et des mâles sauvages est significative $(t=2,57$ d. d. $1 .=7)$ pour ces trois lâchers. Le même test appliqué entre tous les lâchers de mâles stériles et les trois lâchers de mâles sauvages est très significatif $(\mathrm{t}=6,40$; d. d. $1 .=50$ ).

La longévité maximale est donc nettement plus faible chez les mâles stériles que chez les mâles sauvages. Elle est toutefois meilleure que celle observée chez $G$. tachinoides au Tchad (20,3 jours).

La dose de 15000 rads appliquée dans cette expérience est certainement élevée. A la suite des essais récents d'irradiation, il semble que des doses plus faibles devraient être acceptables. On peut espérer alors une amélioration des durées de survie, facteur favorable à une meilleure compétitivité des mâles stériles lâchés.

\section{Contrôle de la fertilité des femelles sau- vages}

Le bois de San devant être libéré au début du mois de décembre, une récolte systématique des femelles sauvages de ce gîte est entreprise. Elle vise à capturer le plus grand nombre possible de femelles afin de mettre ces dernières en observation au laboratoire et de suivre leur potentiel de reproduction. Deux lots ont ainsi été constitués, l'un provenant du lieu d'expérience (bois de San), l'autre d'une galerie forestière n'ayant subi aucune intervention (la Guenako).

\subsection{Lot d'expérience}

46 femelles sont prises dans le bois de San à l'aide du piège biconique au cours de quatre séances du 21 au 29-11-75. Après stockage au laboratoire en salle climatisée, 11 sont mortes des suites de la capture et du transport.

C'est donc un total de 35 femelles qui est mis en observation, chacune d'elles étant placée individuellement dans un bécher fermé par du tulle. Elles sont nourries quotidiennement sur oreilles de lapins. La production de pupes ou d'avortons est enregistrée et, en cas de mort, l'appareil génital femelle est disséqué.

La durée d'observation s'étale sur 46 jours. Les résultats obtenus sont les suivants (tabl. VI).

- 25 femelles produisent au minimum une pupe et au maximum quatre pupes selon leur longévité au laboratoire qui est en moyenne de 31,6 jours $( \pm 5,1)$. Certaines produisent une

\begin{tabular}{|c|c|c|c|c|}
\hline & \multirow{2}{*}{$\begin{array}{l}\text { Nombre de femelles } \\
\text { en observation }\end{array}$} & \multirow{2}{*}{$\begin{array}{c}\text { Nombre de femelles } \\
\text { vierges }\end{array}$} & \multicolumn{2}{|c|}{ Nombre de femelles insêminëes } \\
\hline & & & q fertiles & \& stëriles \\
\hline $\begin{array}{l}\text { Lot expérience } \\
\text { (bois de San) }\end{array}$ & 35 & 1 & 25 & 9 \\
\hline $\begin{array}{l}\text { Lot tëmoin } \\
\text { (Guenako) }\end{array}$ & 30 & 0 & 30 & 0 \\
\hline
\end{tabular}
pupe suivie d'un avorton. Elles sont cependant considérées comme fertiles.

La présence de cicatrices copulatrices est générale.

TABL. $\mathbb{N}^{\circ}$ VI-Fertilitê comparée des femelles sauvages du gîte d'expërience et du gîte têmoin. 
L'utérus est vide dans 12 cas et renferme une larve plus ou moins développée dans 13 cas.

Les spermathèques sont pleines dans 20 cas et à moitié pleines dans 5 cas.

-1 femelle est vierge (absence de cicatrices copulatrices, spermathèques vides).

- 9 femelles ne produisent ni pupe, ni avorton.

La présence de cicatrices copulatrices est générale.

L'utérus est vide dans 7 cas et renferme une larve dans 2 cas. Ces larves n'ayant pas été expulsées au bout de 23 jours pour l'une et 46 jours pour l'autre, sont considérées comme anormales chez des femelles dont l'âge physiologique est de 60-62 jours au moment de la dissection.

En résumé, on retient que 9 femelles sur 34 ne donnent aucune pupe au bout de la durée d'observation: $26,4 \mathrm{p} .100$ des femelles sauvages provenant du gîte d'expérience sont demeurées stériles.

\subsection{Lot témoin}

Celui-ci est constitué de $\mathbf{3 0}$ femelles sauvages capturées sur une autre galerie forestière (bordant la Guenako) à $60 \mathrm{~km}$ de Bobo-Dioulasso.

Ces dernières sont mises individuellement dans des béchers stockés dans les mêmes conditions climatiques et sont nourries avec la même régularité. La durée d'observation est également de 46 jours. 30 femelles produisent durant une longévité moyenne de 32,1 jours $( \pm 3,6)$ de 1 à 4 pupes ou avortons et sont considérées comme pleinement fertiles, l'expulsion d'avortons étant assez communément observée chez certaines femelles après la capture et le transport en cage.

100 p. 100 des femelles sauvages issues d'un autre gite considéré comme témoin sont donc fertiles.

\subsection{Comparaison des deux lots}

- La comparaison entre les pourcentages de fertilité des deux lots est significativement différente $\left(X^{2}=3,89 ;\right.$ d. d. $\left.1 .=1\right)$. Il y a donc eu effet des mâles stériles qui induisent, durant cette période de lâcher, une stérilité de plus de 25 p. 100 dans la population femelle du gîte receveur.

- A quelques exceptions près, les femelles capturées au piège biconique sont des pares (groupe d'âge IV à VII). Les échantillons ainsi récoltés sont constitués d'individus plus âgés que ceux pris par les captureurs (A. CHALLIER, C. LAVEISSIERE) et de ce fait, les femelles mises en observation au laboratoire doivent donner des pupes dans un délai bref après leur capture.

La durée entre le jour de capture et le moment de la larviposition au laboratoire est de 15,1 jours $( \pm 2,6)$ pour le lot d'expérience et de 12,1 jours $( \pm 1,7)$ pour le lot témoin ; elle est significativement différente $(t=2,03 ; \mathrm{d}$. d. $1 .=47)$ ce qui tend à montrer qu'à âge égal, les femelles du gite d'expérience ont une larviposition retardée par rapport à celles du gîte témoin.

- Irradiés à fortes doses (15000 rads), les mâles de G. p. gambiensis sont totalement stériles mais leur compétitivité sexuelle semble amoindrie; le pouvoir d'insémination est plus réduit : 20 p. 100 de spermathèques demi-pleines pour celles qui sont fertiles contre 55 p. 100 pour celles qui sont stériles.

- La présence de larves dans l'utérus de deux femelles âgées qui n'ont cependant produit aucune pupe pendant une longue durée d'observation laisse penser que la viabilité de ces dernières est peu probable et qu'à côté de la stérilité engendrée par les mâles irradiés peut apparaître aussi une mortalité larvaire (tares génétiques ?).

- L'examen, après dissection, des ovaires ne montre pratiquement pas d'anomalies dans le lot stérile. Une seule femelle présente deux gros follicules, un dans chaque ovaire, entraînant une obstruction mécanique.

\section{DISCUSSION}

La compétitivité demeurant l'objectif majeur, on peut essayer de distinguer ce qui a favorisé ou défavorisé les mâles stériles dans cette expérience.

\section{Facteurs favorables}

- La durée du transport et le temps séparant le moment d'irradiation de celui du lâcher sont raccourcis au maximum.

L'ensemble réduit les risques d'usure des ailes des mâles stériles stockés dans les cages, ce qui explique ce faible taux de pertes au moment de l'envol. 
- Le gîte du bois de San a une superficie réduite et son isolement est exceptionnellement bon; les chances de rencontre des mâles stériles et des femelles sauvages sont augmentées et tout accroissement de la population sauvage par apport extérieur est impossible.

- La saison des pluies favorise l'adaptation des mâles stériles aux conditions naturelles (forte humidité, température modérée) ce qui explique les bonnes longévités observées et la dispersion rapide dans le gîte.

\section{Facteurs défavorables}

- La forte dose d'irradiation est certainement le facteur le plus préjudiciable. Si la stérilité obtenue est totale, le pouvoir inséminant reste moindre et l'efficacité souhaitée est de ce fait réduite.

- Les quantités de mâles stériles disponibles sont demeurées relativement faibles et le rapport $\varnothing \rightarrow / \delta^{\wedge}$ n'a atteint en moyenne qu'une valeur de 1 . Supérieur à 2 en fin d'expérience, il reste insuffisant puisqu'il devrait être au minimum de 3 suivant les calculs théoriques (10).

- En dernier lieu, on note qu'en saison des pluies la densité des populations naturelles de G. p. gambiensis augmente notablement. Les mâles stériles ont donc dû affronter une population qui croissait à cette époque, ce qui n'a pas permis d'obtenir un rapport de nombre plus en leur faveur.

Si les mâles stériles ont été favorisés sur certains points, d'autres leur ont été préjudiciables. Un bilan des facteurs favorables et défavorables laisse penser que la compétitivité engagée s'est effectuée dans des conditions moyennes de difficulté pour les mâles stériles.

\section{CONCLUSION}

Durant la saison des pluies 1975, le surplus de mâles produit au laboratoire de l'I. E. M. V. T. à Bobo-Dioulasso a été lâché, après irradiation, dans un gîte réduit et isolé de Glossina palpalis gambiensis.

Plus de 5500 mâles sont ainsi libérés. Leur comportement à l'envol, leur longévité et leur dispersion sont satisfaisants dans des conditions de compétitivité moyennement difficiles. Cette observation limitée indique, qu'avec un rapport de $1 / 1$, les mâles d'élevage irradiés à $15000 \mathrm{rads}$ ont provoqué en quatre mois de lâcher la stérilité d'une femelle sauvage sur quatre. On peut alors penser qu'un rapport d'au moins $4 / 1$ en faveur des mâles stériles soit nécessaire pour rendre stérile toute la population de femelles sauvages d'un gîte isolé. Le rapport devra être amené à un niveau plus élevé encore si l'environnement est moins favorable aux mâles stériles (mauvais isolement du gîte, forte densité, grande durée de transport, etc...).

Ces essais préliminaires ont permis, d'une part de mettre à profit des quantités de mâles produits au laboratoire en zone tropicale pour étudier le comportement sur le terrain des individus stérilisés de l'espèce $G$. p. gambiensis, d'autre part de mettre à l'épreuve matériel et personnel avant une expérimentation très prochaine de la méthode du mâle stérile à plus grande échelle.

\section{SUMMARY}

Preliminary observations on releases of sterilized Glossina palpalis gambiensis (Upper Volta)

During the wet season of 1975,5556 males of G. p. gambiensis irradiated with 15000 rads were released, in 25 lots during a four month period, into a sacred forest close to Bobo Dioulasso. Losses durıng shipment to the release site were low (6.7 per cent). Regular fly samples collected by means of " Challier $»$ tsetse traps, indicated a ratio of one wild to one released male during the observation period, but this ratio fluctuated according to the numbers of flies released and to fluctuations in the density of the wild fly population.

From about the fourth day after release, the dispersal of irradiated males was good. Fifty per cent of the flies died within 6.2 days, but those that survived this period had a mean longevity of 21.2 days. The males thus released, which were in limited numbers and had received a heavy sterilizing dose, had however the effect of preventing 25 per cent of the females captured and kept under observation, from larvipositioning.

This preliminary investigation has permitted studies to be made on the behaviour of sterilized male G. palpals gambiensis when released into the field and to iron out problems of materials and personnel before additional releases are made on a much greater scale. 


\section{RESLMEN}

\section{Observaciones sobre un ensayo preliminar de soltares de machos esteriles} de Glossina palpalis gambiensis (Alta-Volta)

Durante la estación de las lluvias 1975, 5556 machos de G. p. gambiensis irradiados con 15000 rads han sido soltados durante cuatro meses, de 25 veces, en un bosque sagrado cerca de Bobo-Diulaso.

Las perdidas notadas al momento del vuelo son poco importantes $(6,7$ p. 100). Observaciones regulares mediante la trampa Challier indican una razón media de 1 macho esteril para 1 macho salvaje durante el periodo variando según las cantidades soltadas y según la evolución de la densidad natural del sitio de descanso.

La dispersión es buena a partır del cuarto día siguiendo el soltar y la longevidad 50 p. 100 es de 6,2 dias mientras que la longevidad anotada es de 21,2 días por termino medio.

Sin embargo, los machos así soltados, cuyo número está limitado y que reciben una dosis grande esterilizante, han provocado una ausencia de larviposición en 25 p. 100 de las hembras salvajes observadas después de captura.

Este ensayo preliminar ha permitido estudiar el comportamiento de machos esteriles de la especie $G$. palpalis gambiensis soltados sobre terreno y experimentar material y personal antes proximos soltares en gran escala.

\section{BIBLIOGRAPHIE}

1. CHALLIER (A.), LAVEISSIERE (C.). Un nouveau piège pour la capture des glossines (Glossina : Diptera-Muscidae), description et essais sur le terrain. Cah. O.R.S.T. O.M., sér. Ent. méd. Parasit., 1973,9 (4) : 251-262.

2. CLAIR (M.). Projet de lutte contre les mouches tsé-tsé par lâchers de mâles stériles. Rev. Elev. Méd. vét. Pays trop., suppl. pp. 127-129. (Les moyens de lutte contre les trypanosomes et leurs vecteurs. Actes du colloque, Paris, 12-15 mars 1974.)

3. CUISANCE (D.), ITARD (J.). Comportement de mâles stériles de Glossina tachinoides West. lâchés dans les conditions naturelles, environs de FortLamy (Tchad). I. Transport, lâchers, rythme d'activité, action sur la population sauvage. Rev. Elev. Méd. vét. Pays trop., 1973, 26 (1) : 55-76.

4. CUISANCE (D.), ITARD (J.). Comportement de mâles stériles de Glossina tachinoides West. lâchés dans les conditions naturelles, environs de FortLamy (Tchad). II. Longévité et dispersion. Rev. Elev. Méd. vét. Pajs trop., 1973, 26 (2) : 169-186.

5. CUISANCE (D.), ITARD (J.), Comportement de mâles stériles de Glossina tachinoides West. lâchés dans les conditions naturelles, environs de FortLamy (Tchad). III. Lieux et hauteurs de repos. Comportement alimentaire. Rev. Elev. Méd. vét. Pays trop., 1973, 26 (3) : 323-338.

6. CUISANCE (D.), ITARD (J.). Lâchers de mâles stériles de Glossina tachinoides West. dans un gîte naturel de faible densité (Bas-Logone, Cameroun).
Rev. Elev. Méd. vét. Pays trop., 1973, 26 (4) : $405-$ 422.

7. ITARD (J.). Elevage, cytogénétique et spermatogénèse des insectes du genre Glossina. Stérılisation des mâles par irradiation gamma. Ann. Parasit. hum. comp., 1971,46 ( 3 bis $)$ : 35-66.

8. ITARD (J.). Technique d'élevage des glossines. Perspectives offertes pour l'utilisation de la méthode de lutte par lâchers de mâles stériles. Bull. Off. int. Epiz., 1971, 76 : 307-318.

9. ITARD (J.). L'élevage de Glossina palpalis gambiensis Vanderplank, 1949 (Diptera-Muscidae) à MaisonsAlfort. Rev. Elev. Méd. vét. Pays trop., 1976, 29 (1) : 43-58.

10. KNIPLING (E. F.). The potential role of the sterility method for insect population control with special reference to combining this method with conventional methods. U. S. D. A., ARS, 1964, $33-98,54 \mathrm{p}$.

11. LAFAYE (A.). Analyse statistique $\pi^{0}$ 6130. Document technique, O.C. C. G. E., 1976.

12. LANGLEY (P. A.), JORDAN (A. M.). Control by genetic methods. Rev. Elev. Méd. vét. Pays trop., suppl. pp. 137-139. (Les moyens de lutte contre les trypanosomes et leurs vecteurs. Actes du colloque, Paris, 12-15 mars 1974).

13. POLITZAR (H.). Biologische Bekämpfung der Tsetse fliege. $3^{\mathrm{e}}$ conférence Outre-Mer des vétérinaires de la Coopération Technique Allemande. Ouagadougou, 11-19 novembre 1975 (à paraître). 